\title{
El Psicoanálisis en los Inicios de la Relación Psicología-Odontología en la Argentina
}

\author{
Psicanálise no Início da Relação Psicologia-Odontologia na Argentina
}

Psychoanalysis at the Beginning of the Psychology-Dentistry Relationship in argentina

\author{
Patricia Scherman ${ }^{1}$ \\ María Silvia Giordano \\ Universidad Nacional de Córdoba
}

\begin{abstract}
Resumen
En el marco del estudio histórico acerca de las diferentes articulaciones de la psicología con las ciencias de la salud, se investigan las primeras vinculaciones de la psicología con la disciplina odontológica en la Argentina, específicamente con la odontología infantil. Se revisan las producciones elaboradas entre los años 1950 y 1970, y se señala su incidencia en la formación profesional del odontólogo. Se efectuó un estudio historiográfico, basado en el análisis de fuentes primarias, orales y documentales. Los resultados obtenidos muestran que uno de los primeros efectos de esta vinculación fue la contribución a la reformulación de las matrices de comprensión de los odontopediatras sobre la atención del niño. Se señala el importante papel del psicoanálisis en esta reformulación, y se describe como, en la experiencia desarrollada por Arminda Aberastury y María Inés Egozcue, el psicoanálisis y la odontopediatría forjaron, durante ese período, una relación de mutuo beneficio.
\end{abstract}

Palabras clave: Psicología, psicoanálisis, odontopediatría, historia, Argentina

\section{Resumo}

No estudo histórico sobre as diferentes articulações da psicologia com as ciências da saúde, investigam-se os primeiros vínculos da psicologia com a disciplina odontológica na Argentina, especificamente com a odontologia infantil. As produções elaboradas entre os anos 1950 e 1970 são revisadas, e nota-se sua incidência na formação profissional do dentista. Um estudo historiográfico foi conduzido, como base na análise de fontes primárias, orais e documentais. Os resultados obtidos mostram que um dos primeiros efeitos desse vínculo foi a contribuição para a reformulação das matrizes de compreensão dos dentistas pediátricos sobre a atenção da criança. Destaca-se o importante papel da psicanálise nessa reformulação, e descreve-se como, na experiência desenvolvida por Arminda Aberastury e María Inés Egozcue, a psicanálise e a odontopediatria estabeleceram uma relação mutuamente benéfica nesse período.

Palavras-chave: Psicologia, psicanálise, odontopediatria, história, Argentina

\begin{abstract}
In the framework of the historical study about the different articulations of psychology with health sciences, the first links of psychology with dental discipline in Argentina, specifically with child dentistry, are investigated. The productions elaborated between the years 1950 and 1970 are reviewed, and it is noted their incidence in the professional training of the dentist. A historiographic study was conducted, supported by primary, oral, and documentary sources. The results obtained show that one of the first effects of this link was the contribution to the reformulation of the pediatric dentists' understanding matrices on child care. The significant role of psychoanalysis in this reformulation is pointed out, and it is described how, in the experience developed by Arminda Aberastury and María Inés Egozcue, psychoanalysis and pediatric dentistry established a mutually beneficial relationship during this period.
\end{abstract}

Keywords: Psychology, psychoanalysis, pediatric dentistry, history, Argentina

\footnotetext{
${ }^{1}$ Dirección: Bv. de la Reforma esquina Enfermera Gordillo, Ciudad Universitaria, s/n, Córdoba, Argentina, CP 5000. E-mail: patoscherman@gmail.com
} 


\section{Introducción: La Expansión del Psicoanálisis y los Inicios de la Relación Psicología-Odontología}

La psicología en la Argentina se ha situado desde sus orígenes en un entrecruzamiento de influencias de diferentes corrientes y orientaciones de la psicología, con particular influencia del psicoanálisis (Ardila, 1979; Vezzetti, 1996a; Klappenbach, 2006; Piñeda \& JacóVilela, 2014). Se trata de una disciplina de constitución compleja, que junto a otras ciencias integra un campo con diversidad de problemas teóricos y metodológicos (Falcone 2010; García, Macchioli \& Talak, 2014; Vezzetti, 2007). La influencia del psicoanálisis ha otorgado una importante dosis de originalidad al rol profesional del psicólogo argentino, y también a las carreras de Psicología del país (Carpintero \& Vainer, 2004; Falcone 2010; Vezzetti, 1996a). Esta influencia, si bien ha gozado de amplia aceptación, no siempre ha sido sido favorable para la instauración de un rol profesional diverso (Vilanova, 2003; Ardila, 1979; Piñeda \& Jacó Vilela, 2014).

El inicio de la colaboración interdisciplinaria de la psicología, y particularmente del psicoanálisis, con la odontología comenzó a desarrollarse a principios de los años 1950 y se mantuvo firme hasta principios de la década de 1970. El evento que nos permite fechar el inicio de esta colaboración es la publicación, en la Revista de Odontología de la Asociación Odontológica Argentina, del artículo de Arminda Aberastury titulado Trastornos emocionales en el niño vinculados a la dentición, en 1951. Este vínculo entre una entidad profesional como la Asociación Odontológica Argentina (AOA) y el psicoanálisis se extenderá luego al ámbito oficial, a la Facultad de Odontología de la Universidad de Buenos Aires, donde la odontóloga María Inés Egozcue y la psicoanalista Arminda Aberastury iniciaron una experiencia de trabajo conjunto en las salas odontopediátricas.

El saber adquirido en estas experiencias colaborativas fue plasmado en tres importantes textos, El psicoanálisis de niños (Aberastury, 1972), Aportaciones al psicoanálisis de niños (Aberastury, 1972) y Odontopediatría y Psicología (Egozcue, Manfredi, \& Basso, 1988) que analizaremos a continuación.

La segunda vía de vinculación entre una psicología con influencia psicoanalítica, pero más amplia que este, y la odontología, se produjo mediante la formación de posgrado de los odontólogos, a través de los cursos de especialización en odontopediatría brindados por la Asociación Odontológica Argentina (AOA) en Buenos Aires, la capital del país.

Estas primeras formas de vinculación nos permitirán reconocer la importancia del psicoanálisis y la psicología en la constitución del campo profesional odontopediátrico. También el papel de la capital del país como centro intelectual, y el de las asociaciones profesionales - tal como la AOA- en la articulación de saberes y prácticas. Años más adelante, luego de un período de fortalecimiento de la profesión del psicólogo y los saberes asociados a la psicología (Klappenbach, 2006), esta vía de relación cambiará. La capacitación en psicología se integrará a la formación de grado del odontólogo, con la inserción de la asignatura Psicología Evolutiva en el segundo año de la carrera de Odontología (UNC, 1985). A partir de esa nueva forma de institucionalización de la psicología, aparecerán nuevas articulaciones, ya sin la participación de las asociaciones profesionales ni de la capital del país como centro intelectual, con un franco declive de la influencia del psicoanálisis en la actualidad (Moncunill de Chaer, 2012; Giordano, 2018). 
Los objetivos de esta presentación consisten en: 1) Reconocer los aportes del psicoanálisis y la psicología en la formación profesional de odontólogos en la Argentina, en las décadas de 1950-1960; 2) Identificar las vías por las que esta influencia se plasmó, en los inicios de la formación profesional de odontólogos, tanto en el grado como en el posgrado; 3) Caracterizar el modelo de intervención sobre el niño que se puso en juego en la experiencia conjunta entre psicoanalistas y odontólogos en la Universidad de Buenos Aires en dicho período, y finalmente 4) Comprender mejor una de las maneras por la cual el psicoanálisis logró imponerse como tecnología de intervención y saber experto en temas de la infancia en la Argentina en la década de 1960.

\section{Metodología}

Esta indagación se configura como un estudio historiográfico, asentado en el análisis de fuentes primarias, orales y documentales (Klappenbach, 2014; Jacó-Vilela, do Espírito-Santo, Degani-Carneiro, de Oliveira, \& Vasconcellos, 2016). Entre las primeras, se analizan una serie de entrevistas realizadas a una reconocida odontopediatra de la época, que participó en las experiencias analizadas- Dra. Perla Krupnik de Hidalgo, profesora emérita de la Facultad de Odontología de la Universidad Nacional de Córdoba (UNC). El análisis de las entrevistas se realizó en base a una codificación textual, primero abierta y luego temática (Flick, 2004; Gibbs, 2012), según las siguientes categorías: el niño como paciente, la experiencia de formación en Buenos Aires y la relación Odontopediatría-Psicología (Giordano \& Alvárez, 2016).

El relevamiento documental descansó en dos tipos de fuentes: los archivos oficiales de la Universidad Nacional de Córdoba y los textos publicados por Arminda Aberastury en los años 1951, 1971 y 1972. De los primeros, se realizó un análisis de contenido de la ordenanza de creación de la Facultad de Odontología de la Universidad Nacional de Córdoba (UNC, 1956), el programa de la materia de Odontología Infantil del año 1958 (Gacioppo, 1958), y el plan de estudios de la carrera de Odontología (UNC, 1985). Entre los segundos, se examinaron dos textos de Arminda Aberastury en los cuales se sintetizaba la experiencia en estudio: El psicoanálisis de niños (Aberastury, 1972) y Aportaciones al psicoanálisis de niños (Aberastury, 1972). Este último incluye un capítulo escrito por la odontopediatra María Inés Egozcue, el cual se incluye en el análisis. A fin de complementar la información recabada, se recurrió al texto de María Inés Egozcue Odontopediatría y Psicología (Egozcue et al., 1988), que, si bien fue publicado años más adelante, expone detalladamente las experiencias de trabajo realizadas en los años 1960. El texto de Egozcue et al., (1988), permite apreciar las actividades realizadas en el marco de la cátedra de Odontopediatría, desde el punto de vista de los odontólogos. El análisis del contenido efectuado se mantuvo en un nivel descriptivo (Rosa, Huertas y Blanco, 1996; Vallés, 1999) limitado a la caracterización de las técnicas construidas en ese espacio colaborativo, en nuestro período temporal.

Considerados como documentos históricos, el análisis de los textos de Aberastury se realizó en base a las pautas de análisis de discurso del material histórico (Rosa et al., 1996), de acuerdo con tres momentos: descripción-contenido, estructura y lenguaje empleado-, explicación e interpretación del mismo. Se buscó identificar la forma expresiva del texto, la función social que cumplió, su significado en la cultura, y finalmente señalar la heurística positiva del texto, la que nunca es definitiva. El estudio de los textos como productos históricos 
resulta valioso, ya que además de proporcionar la base material para el análisis, nos pone en contacto con las formas de circulación de los saberes de una época.

La presentación se organiza en tres partes: en primer lugar se presentan las características del psicoanálisis argentino y en particular del kleinismo como matriz de comprensión, que permitió iniciar una articulación entre odontopediatría y psicoanálisis; luego se exponen las características del trabajo conjunto entre psicoanalistas y odontólogos, la expansión de esas nuevas concepciones al interior del país mediante la formación profesional de odontólogos; y finalmente se revisa cómo el psicoanálisis logró imponerse como tecnología de intervención y saber experto en temas de la infancia.

\section{Odontopediatría y Psicoanálisis: El Kleinismo como Matriz de Comprensión}

Hacia fines de la década de 1930 el psicoanálisis había comenzado a ser visto en la Argentina como una herramienta para la modernización de la psiquiatría y también como un instrumento para la crítica social (Plotkin, 2003). Se presentaba como una disciplina autónoma, tanto por sus bases teóricas y metodológicas, como por la relevancia de sus objetos y temas. Esta relevancia se proyectaba como una disciplina que acompañaba el proceso de modernización de la sociedad (Vezzetti, 1996b). La psicología y el psicoanálisis se presentaban como disciplinas capaces de intervenir y dar respuesta a un amplio abanico de problemas de la personalidad y la familia. Desde el punto de vista social el psicoanálisis armonizaba con este nuevo clima de ideas, venía a ocupar el lugar vacante de ciencia moderna capaz de dar respuestas a las demandas de una sociedad en transformación, que contaba con herramientas teórica y tecnológicas para conocer el alma humana (Plotkin, 2003). Por su parte, la psicología profesional se encontraba en un momento de desarrollo muy incipiente. Las carreras de psicología en el país se crearon a partir de la segunda mitad la década de 1950, y los primeros psicólogos diplomados aparecieron recién en la década de 1960. En esta primera etapa, la psicología argentina se encontraba dominada por la influencia del psicoanálisis, tanto que se ha denominado este período como el de la psicología psicoanalítica (Ardila, 2004; Klappenbach, 2006).

Se ha considerado que la temprana institucionalización del psicoanálisis en la Argentina sentó las bases de su desarrollo ulterior y posibilitó su crecimiento como saber técnico específico, así como su expansión cultural (Dagfal, 2009; Plotkin, 2003; Scherman, 2014). Fundada en Buenos Aires en el año 1942, la Asociación Psicoanalítica Argentina fue autorizada a funcionar de manera provisoria desde su creación, y reconocida como filial de la International Psychoanalytic Association (IPA) en 1949. Esto fue posible gracias a que cumplía uno de los requisitos y estándares establecidos por la IPA más difíciles de satisfacer en estas latitudes: la existencia de analistas didactas que pudieran formar nuevos psicoanalistas. Tres de sus miembros fundadores, Ángel Garma, Celes Cárcamo y Marie Langer, habían concluido sus análisis en Europa y podían ocuparse de la formación de los futuros analistas.

Entonces, hacia los años 1950, el ambiente intelectual de modernización comenzaba a dejar de lado las explicaciones fenomenológicas y existencialistas de posguerra, y requería de modelos teóricos, que, como el psicoanálisis, proponían una articulación más accesible entre sus postulados teóricos y la intervención técnica (Neiburg \& Plotkin, 2004). En el campo más amplio de las ciencias de la salud, resultó de suma importancia la inserción de los 
psicoanalistas allí, Arnaldo Rascovsky en pediatría, Enrique Pichon Riviere en psiquiatría, y Arminda Aberastury, esposa de E. Pichon Riviere, como promotora del psicoanálisis de niños.

La dominante general del psicoanálisis argentino por aquellas épocas estaba representada por el saber freudiano desde la óptica de la escuela inglesa. Se reconoce a la perspectiva kleiniana como una marca distintiva del movimiento psicoanalítico argentino y se la considera como uno de los ingredientes primordiales en la construcción de los discursos psicológicos en la Argentina durante la segunda mitad del siglo XX (Dagfal, 2009).

En el caso de Arminda Aberastury, si bien ella misma reconoció una influencia temprana de Anna Freud, se ocupó de explicitar su adhesión a las teorías de Melanie Klein. Esta adhesión se vio fortalecida a partir del intercambio que sostuvo con dicha autora a propósito de la traducción que efectuara al español, del texto El psicoanálisis de niños (Klein,1944) y de la que luego tomó alguna distancia al producir conceptos propios (Bloj 2009; Dagfal, 2009). Según Arminda lo ha relatado, el dominio de la perspectiva kleiniana le permitió mayor libertad en el desarrollo de muchos de sus postulados teóricos y técnicos ulteriores (Aberastury, 1971, p. 10). Recordemos que en la discusión entre M. Klein y A. Freud intervenían varios vectores (Likierman, 1995; Delahanty, 2003), entre los que podemos destacar el momento de formación del superyó en el niño, la pulsión de muerte, el alcance del psicoanálisis infantil, las modalidades de interpretación en el espacio terapéutico, el trabajo con la transferencia negativa. Para Anna Freud la sublimación de conflictos inconscientes y el aspecto pedagógico del tratamiento infantil son elementos de importancia. Para Melanie Klein, educar y analizar son dos posiciones opuestas e incompatibles; para esta autora el juego infantil es susceptible de ser interpretado, ya que lo concibe como un espacio de representación de las fantasías inconscientes. Para ello se provee al niño de una caja de juegos, a través del cual el niño arma una escena que brinda acceso a su mundo fantasmático. Melanie Klein afirma la importancia del primer año de vida y de las primeras experiencias del lactante en la relación de objeto y el manejo de las ansiedades persecutorias, de allí la importancia de la primera dentición y la primera articulación con la odontología.

La influencia kleiniana es visible en el artículo Trastornos emocionales en el niño vinculados a la dentición (Aberastury de Pichon-Rivière, 1951). Allí Arminda expone su concepción acerca de dicho fenómeno e indica cuáles son los hechos en la fase previa a la dentición que pueden contribuir a formar síntomas. Realiza recomendaciones acerca de las medidas que pueden tomarse para prevenir la acumulación de ansiedad y agresión en el niño para una dentición sin trastornos. En 1971 agregará a este cuadro teórico, la importancia de las ansiedades de los padres frente al tratamiento odontológico de sus hijos y el papel que estas juegan en el mismo.

En la década de 1970, luego de haber desarrollado las experiencias de trabajo conjunto entre odontólogos y psicoanalistas en las salas de Odontopediatría de la UBA, y por ende, de haber tratado específicamente las ansiedades de los niños frente a la experiencia odontológica, declarará su satisfacción por haber podido confirmar las hipótesis de los años 1950, sobre la existencia de ansiedades subyacentes durante el tratamiento odontológico en niños (Aberastury, 1971). 


\section{El Trabajo Conjunto entre Psicoanalistas y Odontólogos}

La producción de Aberastury respecto del campo odontopediátrico se expresó claramente en los textos mencionados. En el primero, Aportaciones al psicoanálisis de niños (Aberastury, 1971), plantea las bases teóricas-técnicas que orientaron al grupo de odontopediatras y psicoanalistas (Aberastury, 1971), y en el otro se enfoca en las posibles aplicaciones del psicoanálisis en el área de la salud (Aberastury, 1972).

En el primero se señala la trascendencia del acercamiento con el campo odontológico: "Creo no equivocarme si señalo que fue en mi país donde por primera vez los odontopediatras pidieron información y se acercaron al análisis" (Aberastury, 1971, p. 11). Se destaca también el valor de la experiencia conjunta, como una manera empírica de comprobar los postulados psicoanalíticos (Aberastury, 1971; Winkler \& Wolff Reyes, 2005). Se indica también, la importancia de la técnica de grupos con padres y madres implementadas en las experiencias conjuntas y en el tratamiento grupal con niños y adolescentes:

La combinación de psicoterapia en grupo para padres y para niños,-realizada por terapeutas diferentes- ha dado excelentes resultados en aquellos niños que presentaban ansiedades que hacían imposible el tratamiento odontológico. Los fundamentos de la técnica son psicoanalíticos pero la técnica misma es el resultado del esfuerzo de un equipo de odontopediatras que trabajaron junto a psicoanalistas de niños (Aberastury, 1971, p. 9).

En este texto se reproduce el artículo inicial, Trastornos emocionales en el niño vinculados con la dentición (Aberastury de Pichon-Riviére, 1951), y aparece también un llamativo capítulo escrito por la odontopediatra María Inés Egozcue denominado Aportes psicoanalíticos a la clínica odontopediátrica. En él se observa una importante utilización de conceptos teóricos y de lenguaje psicoanalítico que da cuenta de una sólida apropiación del psicoanálisis por parte de la odontopediatra.

En el segundo texto, El psicoanálisis de niños y sus aplicaciones (Aberastury, 1972), la autora expone las aplicaciones de su técnica en la preparación psicoterapéutica del niño que debe someterse a cirugía, y luego se refiere específicamente a la psicoterapia en la práctica odontopediátrica. En la tercera parte reúne colaboraciones de psicoanalistas, psicólogos y asistentes sociales sobre la psicoterapia del grupo familiar con niños. Además de la revisión de conceptos sobre la dentición en Freud, Abraham y Klein, aparecen sus propias ideas sobre dentición en relación con la genitalidad temprana. En ese sentido, el psicoanálisis de niños aportó a la comprensión del significado de la ansiedad manifestada por los niños, que aparecía como desproporcionada e inmanejable en muchos tratamientos odontológicos.

Las experiencias que posibilitaron estos textos se efectuaron en la Facultad de Odontología de la Universidad de Buenos Aires, desde el año 1959 hasta 1968, en la cátedra de Odontopediatría, cuya profesora titular era la Dra. María Inés Egozcue. De larga y reconocida trayectoria en el campo odontopediátrico argentino, la Dra. Egozcue llegó a ser Profesora Emérita de la Universidad de Buenos Aires y Consejera de Posgrado en la Asociación Odontológica Argentina (Bordoni, 2009). Su formación en Odontopediatría se inició en Estados Unidos, en Michigan, con Kenneth Easlick, pionero de la odontología infantil. Easlick había publicado en 1935 el célebre artículo The dentist's management of young 
children (Easlick, 1935), donde señalaba la importancia de la psicología para la profesión odontológica, indicando que ésta tiene una "necesidad crónica" de ser asistida por la psicología infantil (Easlick, 1935, p. 1). Entre las bases psicológicas presentes en este artículo encontramos dos citas directas de J. B. Watson (Watson, 1928; 1929). Egozcue recibió de Easlick la idea de prestar atención a la conducta del niño para el desarrollo exitoso de la consulta odontológica (Bordoni, 2009). Esta matriz de formación, que incluía los aspectos conductuales del niño, incorporó luego el importante aporte realizado por Arnold Gesell (Gesell, 1943). Inicialmente, esta fue la línea teórica que comenzó a desarrollarse en los cursos de posgrado en la Asociación Odontológica Argentina desde 1948; el conocimiento del niño se basaba en los textos de Gesell, con aportes de Piaget, Bühler y Spitz (Bordoni, 2009; Krupnik de Hidalgo, comunicación personal, 2016).

No obstante, la importancia reconocida de estos aportes, en la Argentina comenzaban a aparecer demandas de nuevos conocimientos, que permitieran una mejor atención de los niños en el espacio específico de la consulta odontológica. Un relato de Egozcue muestra nítidamente las dificultades en que se encontraban estas pioneras en su campo:

Pese al gran avance que se había dado, ese niño comprendido con criterio evolutivo no siempre permitía su manejo en el sillón. Un ejemplo de la práctica diaria aclarará algunas de las dificultades en que se encontraba la profesión. El odontólogo sabía, de acuerdo con los principios gesellianos, que un niño de cinco años tiene una conducta armoniosa, está en buenas condiciones consigo mismo, el hogar y el ambiente cultural que lo rodea y era esperado en los consultorios con tranquilidad. Llegaban a los mismos muchos niños de cinco años normales pero que en nada recordaban las plácidas características de su edad. Se presentaban huraños, agresivos, de difícil comunicación y, muy a menudo, con un pasado médico y odontológico psíquicamente traumático. Para encarar su atención no alcanzaban los conocimientos que la profesión poseía. ¿Qué hacer? (Egozcue como se cita en Aberastury, 1972, p. 154).

La sensación de insuficiencia de los saberes adquiridos, en un ambiente de recepción favorable a las ideas psicoanalíticas (Plotkin 2003, Dagfal, 2009), dieron lugar a la implementación de una experiencia institucional de trabajo, denominada luego Tratamiento Combinado Odontológico-Psicológico (Egozcue et al., 1988).

Esta experiencia introdujo grandes cambios en la labor clínica odontopediátrica. El modelo de tratamiento incluía diversas técnicas de acercamiento a la experiencia odontológica: cambios en la sala de espera compartida con adultos, recepción del niño con su madre, atención al historial del primer año de vida del niño y de sus circunstancias médicas vividas, a su entorno familiar y ambiente sociocultural-económico. También técnicas para desplazar las fantasías inconscientes traducidas en ansiedades, técnicas de extracción dentaria teniendo en cuenta los conceptos de destrucción-reparación, de esquema corporal, y de clases de duelos, así como la incorporación de la madre como parte del tratamiento y la explicitación del encuadre (Egozcue et al., 1988).

Si bien los resultados de estas experiencias interdisciplinarias buscaron ser consideradas como avances de investigación, -fueron presentadas por la Dra. Egozcue a la Sociedad Argentina de Odontología, a la Internacional Association for Dental Research, al Dr. Houssay, en su calidad de director del CONICET-, la vía de la investigación institucionalizada no 
prosperó para estos desarrollos (Egozcue et al, 1988). No obstante, debido al crecimiento de la actividad psicoterapéutica en la cátedra de Odontopediatría, se logró la asignación de cargos docentes rentados para los psicoterapeutas, quienes se integraron al equipo clínico (Egozcue et al., 1988).

El propósito inicial de la experiencia conjunta era evitar la anestesia general y también realizar la preparación psicológica para la anestesia general. Sin embargo, desde el año 1963 ya no fue necesario realizar anestesia general para las intervenciones odontológicas, ya que se advirtió que con los niños que concurrían al grupo terapéutico no era necesaria dicha práctica (Salas, Forti, Saimovici, Sirota como se cita en Aberastury, 1972).

El modelo de trabajo era de tipo grupal, organizado a partir de los niños que presentaban dificultades para el tratamiento odontológico. Se trabajaba "exclusivamente sobre las fantasías de épocas primarias del desarrollo en relación con ansiedades orales y genitales que se reactivaban frente al tratamiento odontológico" (Aberastury, 1971, p. 123). Se les aclaraba el significado del tratamiento, como una manera de trabajar sobre las resistencias que presentaban, para que pudieran recibir voluntariamente las intervenciones odontológicas necesarias. Desde la perspectiva kleiniana empleada, se consideraba que el emergente que provocaba las ansiedades en los niños era la fantasía de que las piezas dentarias extraídas no fueran repuestas. Se proponía un trabajo de elaboración analítica de esa ansiedad, que permitiera comprender el significado reparador del tratamiento odontológico, y así disminuir el miedo del niño, tanto al tratamiento como a la persona del odontólogo.

El trabajo con estos grupos de niños permitió a los psicoanalistas ajustar la técnica inicial, especialmente al incorporar grupos de madres o padres que acompañaban a los niños a la consulta odontológica. "Se pudo comprobar entonces la estrecha relación existente entre las fantasías orales y genitales y la similitud del material entre el grupo de niños y de padres" (Aberastury, 1971, p. 123).

El modelo de trabajo en equipo incluía la realización de reuniones periódicas entre psicoterapeutas y odontopediatras; allí se establecían las pautas para las entrevistas odontopediátricas, los criterios a seguir con respecto al envío de los niños al grupo terapéutico, tamaño del grupo y alta del grupo, la convocatoria a las madres y los modos de comunicación odontólogo-terapeuta (Egozcue et al., 1988, p. 261).

Participaron de esta experiencia, en diferentes períodos, reconocidos psicoanalistas tales como Susana Lustig de Ferrer, Eduardo Salas, Lidia Forti, Edmundo Saimovici y Alicia Sirota; más adelante, Gela Rosenthal, Moisés Trajtemberg, Jorge Rovatti, Julio Nejamkis, entre otros (Aberastury, 1972; Egozcue et al., 1988). Estos profesionales señalaron que esa experiencia les posibilitó revisar en la práctica el significado de la aparición de dientes en el niño, así como acrecentar su formación en psicoanálisis de niños.

Con relación a las técnicas empleadas, los participantes señalaban que estas se fueron definiendo en terreno en base al esquema referencial psicoanalítico y adaptadas a la situación junto a los odontólogos. Se utilizaba la psicoterapia de grupo para niños y también para madres o grupos de acompañantes, coordinadas por un psicoanalista. Los grupos de niños de entre 2 y 12 años se dividían en preescolares y escolares, y las madres se reunían simultáneamente con otro analista, en otro ambiente, el mismo día y horario que sus hijos, El grupo se reunía dos veces por semana, durante dos meses, funcionando en los horarios de trabajos prácticos de la cátedra. Se trataba de casos graves o difíciles: "Todos ellos tenían un 
estatus odontológico muy comprometido que imponía un promedio de 6 a 8 extracciones, independientemente de los diversos arreglos que igualmente debían efectuarse" (Ferrer como se cita en Egozcue et al., 1988, p. 237). La técnica utilizada era la interpretación psicoanalítica del material de juegos en el plano profundo, característica de la psicoterapia de grupos en niños. A la caja de juegos se incorporó material odontológico específico- pinzas y fórceps para extracciones, así como el espejo de mano- (Egozcue como se cita en Aberastury, 1972); esta incorporación de nuevos elementos a la caja de juegos requería por parte de los terapeutas un conocimiento acabado de las prácticas que debían ejecutarse en la boca de cada niño, la manera en que iban a realizarse y con qué instrumental (Egozcue et al., 1988; Salas, Forti, Saimovici, Sirota, como se cita en Aberastury, 1972). Con las madres, en cambio, se utilizó una técnica distinta, dado que influía "su compromiso con el mundo externo, las defensas más consolidadas y la breve duración de la terapia" (Salas, Forti, Saimovici, Sirota, como se cita en Aberastury, 1972, p. 85)

\section{La AOA y la Formación de Odontopediatras}

La necesidad de los odontopediatras de encontrar aportes que permitieran comprender mejor a este nuevo paciente, y en consecuencia manejar más adecuadamente la situación odontológica con niños, los condujo a incrementar su formación. Se procuraba un mayor dominio de la técnica odontológica específica para el tratamiento de niños y también en el reconocimiento de las particularidades del proceso de desarrollo psicológico, especialmente en lo que atañe a las significaciones que se depositan en el cuerpo del niño y, en particular, en la boca (Egozcue como se cita en Aberastury, 1972; Krupnik de Hidalgo, comunicación personal, 2016).

A principios de los años 1950, esta matriz de comprensión del niño difundida en Buenos Aires comenzó a expandirse hacia el interior del país. En Córdoba, parte de estos conocimientos se incluyeron tempranamente en la formación de odontólogos, en los contenidos de la asignatura Odontología Infantil. Es necesario destacar que la Facultad de Odontología, como institución independiente, se fundó en el año 1956, en el marco de una importante transformación del sistema universitario nacional en la Argentina (Buchbinder, 2005). Junto con la creación de la Facultad, se gestó un nuevo plan de estudios (UNC, 1956), en el cual se incluyó la asignatura Odontología Infantil, cuya profesora titular, Dra. Hermosina Gacioppo, comenzó a incluir temas de psicología en unidades específicas del programa (Gacioppo, 1958). Estos nuevos conocimientos estaban orientados a formar al odontólogo en una mejor comprensión de las características evolutivas del niño, para poder concebirlo como un paciente diferente del adulto, tanto en el aspecto morfológico como en el psíquico. Esta inclusión se instituía como un nuevo ingrediente en la construcción del objeto de la especialidad, y al mismo tiempo les permitía tener en cuenta los requerimientos del niño desde la primera consulta, representaba una importante actualización profesional.

Por aquellos años, los profesionales de todo el país interesados en completar una capacitación específica en niños se dirigían a la capital del país, para cursar los programas de formación de la AOA. La capacitación adquirida en estos cursos les permitiría desempeñarse como odontopediatras y luego participar en la formación de nuevos profesionales en sus lugares de procedencia. Tal fue el caso de las Profesoras Hermosina Brizuela de Gacioppo y Perla Krupnik de Hidalgo, de la Universidad Nacional de Córdoba, quienes se formaron en 
Buenos Aires, en las modernas tendencias de la odontología infantil, y quienes luego incorporaron los contenidos aprendidos en Buenos Aires en los programas de Odontopediatría de la carrera de Odontología (Gacioppo, 1958).

Para analizar las características de la formación adquirida en Buenos Aires, en la AOA, y el papel que ésta desempeñó en la transformación de la atención odontopediátrica, recurrimos a una serie de entrevistas realizadas a una de las participantes de estos procesos, Dra. Perla K. de Hidalgo.

En dichas entrevistas aparece como un rasgo destacado el carácter novedoso que representó la incorporación de estas nuevas concepciones sobre el niño, y el descubrimiento de la importancia de incorporar aportes de la psicología para una mejor atención del niño en la consulta odontológica. De las categorías analizadas, se desprende lo siguiente:

Con relación al niño como paciente, nuestra entrevistada refiere que, en las épocas previas a la creación de la Facultad de Odontología, no existía una consideración específica sobre el paciente niño, no existía cátedra donde se lo abordara, y que, en definitiva, se lo trataba como a un paciente adulto.

Esta situación produjo dos movimientos: la necesidad de acrecentar la formación profesional específica. Y luego, la incorporación de esos conocimientos y esa experiencia de formación en los programas de las materias de la carrera de Odontología, en este caso en Córdoba. Tanto nuestra entrevistada como la profesora Hermosina Gacioppo cursaron el mismo programa de formación de posgrado, lo cual influyó en la idiosincrasia que tomó la cátedra de Odontología Infantil en la UNC.

Con relación a la experiencia Buenos Aires, en la AOA, las profesionales percibían una confluencia disciplinar entre la odontología y la psicología de corte psicoanalítico para el abordaje de los niños. La promoción de este cruce disciplinar se basaba en la convergencia existente entre el desarrollo de la dentición temprana y el desarrollo del psiquismo entendido desde una perspectiva kleiniana.

Con relación al vínculo Odontología y Psicología, este se basó en los lineamientos del programa de formación impartido en Buenos Aires, el cual incluía aspectos de corte evolutivo-comportamental como psicoanalítico. Este entrecruzamiento, que si bien presenta concepciones teóricas y praxiológicas altamente diferenciadas y aún contrapuestas, era recibido por los odontólogos como un aparato conceptual integrado. El profesional tomaba de allí, tanto los aspectos evolutivos como los contextuales y dinámicos de la conducta del niño para resolver la consulta odontológica de una manera que se consideraba moderna.

\section{El Psicoanálisis Como Tecnología de Intervención}

Destacamos en primer lugar, el reconocimiento de la eficacia que la intervención psicoanalítica obtuvo en aquel momento para la aceptación voluntaria del tratamiento odontológico por parte de los niños y la suspensión de la anestesia general como modo de resolver la consulta odontopediátrica. La finalidad de esa intervención no se dirigía a resolver conflictos psicológicos ni modificar estructuras mentales, como en el caso de un psicoanálisis infantil. No obstante, se reconocían efectos más allá de la finalidad primera, dado que la comprensión de la idea de reparación del daño permitía a los niños conducirse mejor frente a los conflictos en general. Asimismo, entre otros efectos beneficiosos de la experiencia se señalaba la mejora en la comunicación entre los niños y sus familias. 
La inclusión de conocimientos psicoanalíticos en el campo de la odontología se inició en la Argentina con la demanda de los odontopediatras y puede encuadrarse bajo el concepto de interés intelectual propuesto por Danziger (1979) en términos de intereses duales, tanto para los psicoanalistas como para los odontólogos. De parte de los odontólogos estos aportes representaban una alternativa para poder solucionar lo que se le presentaba como obstáculo en su práctica, aquello que los profesionales percibían como falta de colaboración del niño o bien sus miedos ante la intervención odontológica. En este movimiento, la psicología y en especial el psicoanálisis se legitimaban como forma de conocimiento y como práctica pertinente ante una ciencia de la salud como es la odontología, donde el énfasis en la comprensión de los niños estaba puesto en la realización exitosa de la intervención clínica.

Hacia el exterior, el interés intelectual básico de una disciplina busca legitimar las actividades de sus practicantes con relación a los grupos significativos a los que se dirige (Danziger, 1979), y hacia el interior, se pudieron establecer las normas por las cuales se juzgaba el trabajo de los practicantes de esa disciplina (Danziger, 1979). Una vez consolidados los fundamentos institucionales de una disciplina, dichas normas adquieren una validez autónoma y el nexo con la función legitimante externa se vuelve menos explícito. De allí el valor de un estudio historiográfico, que devele este proceso de construcción. La historicidad de los objetos de una disciplina se revela, entre otros, en la historia de los cambios de los conceptos de la misma (Danziger, 1997).

En segundo lugar, se produjo un importante efecto de difusión del psicoanálisis, a partir del trabajo con los grupos de madres. Vinculadas de manera indirecta con la resolución de la consulta odontopediátrica, las madres representaron el segundo objeto de intervención. Allí la acción tuvo como efectos difundir las ventajas del abordaje psicoanalítico para atender a los problemas de la niñez. Se buscaba que las madres reconocieran el papel de los adultos en los problemas de los niños, a la vez que incorporaran a esta representación la necesidad de la intervención de un experto para un mejor devenir de la infancia. Por medio del par conceptual elaboración vs. desplazamiento, el psicoanálisis mostraba los riesgos de considerar solucionado el conflicto psiquico una vez resuelta la cuestión odontológica, ya que podría haber desplazamientos hacia otras partes del cuerpo. De allí que, hacia las madres, se enfatizaba la importancia de un enfoque preventivo ante las dificultades, y no orientarse por la simple eliminación de las mismas. Se proponía así, un modelo de comprensión específico de los problemas de la infancia, dado que los terapeutas recomendaban hacer extensiva la técnica empleada a otras situaciones posiblemente traumáticas en los niños como operaciones o viajes.

El efecto de difusión del abordaje psicoanalítico nos remite a la importancia de esa representación que tomó forma en los años 1960. La concepción del papel de la madre en las dificultades del desarrollo de sus hijos gozó de gran pregnancia en la psicología argentina y consolidó un nuevo sentido común (Borinsky, 2006).

Finalmente, consideramos que esta experiencia permitió consolidar la matriz de comprensión kleiniana (Giordano, Scherman \& Álvarez, 2017), ya que al concebir “. . . una organización genital temprana en el lactante permitieron la operatividad de la técnica y la comprensión del material clínico" (Salas, Forti, Saimovici, Sirota como se cita en Aberastury, 1972, p. 85). Esta experiencia permitió a los psicoanalistas un estudio intensivo de los conflictos en el individuo de temprana edad, la realización de ajustes en los conceptos teóricos 
kleinianos acerca conflictos vinculados a la dentición en los niños, y finalmente la adaptación de conocimientos psicoanalíticos a campos nuevos, produciendo innovaciones en la psicoterapia de grupos de niños y madres.

\section{Consideraciones Finales}

El estudio histórico de la psicología en interacción con otra disciplina permitió visualizar los aportes de la psicología y el psicoanálisis en la conceptualización del objeto de esa disciplina, en este caso la odontología infantil.

El análisis de la experiencia realizada en la cátedra de Odontopediatría de la Universidad de Buenos Aires y recogida por Arminda Aberastury y María Inés Egozcue en sendos textos, mostró el establecimiento de una matriz de comprensión elaborada a partir de un problema específico de la práctica clínica y confirmó el papel del psicoanálisis como discurso constituyente de la psicología en la Argentina en las décadas de 1960 y 1970.

Entonces, con relación a los objetivos propuestos en este trabajo, consideramos que se han logrado reconocer los aportes del psicoanálisis y la psicología a la odontología infantil. Además, se han podido identificar las vías por las cuales esta influencia formativa se cristalizó en el período inicial de la formación de odontólogos, primero en el posgrado, gracias a la acción de las asociaciones profesionales, y luego su expansión a la formación de grado, así como difusión hacia el interior del país. Finalmente se ha logrado caracterizar acabadamente el modelo de intervención que se puso en juego en la experiencia conjunta entre psicoanalistas y odontopediatras en la Universidad de Buenos Aires en la década de 1960, el cual estuvo determinado por una perspectiva kleiniana según las adaptaciones realizadas por Arminda Aberastury, en conjunto con María Inés Egozcue y los equipos que las acompañaban.

Estos elementos han permitido comprender mejor una de las maneras por las cuales el psicoanálisis logró imponerse como tecnología de intervención y saber experto en temas de la infancia en la Argentina en la década de 1960. El análisis realizado logra retratar un momento de pleno desarrollo del psicoanálisis argentino, como teoría, como terapéutica y como método de investigación.

Se revela también cómo se fortaleció una representación sobre el papel de la madre las dificultades manifestadas por el niño, representación que se completaba con la instauración de una nueva necesidad, la de acudir a un experto para un desarrollo infantil saludable.

En cuanto a las limitaciones de este estudio histórico, la imposibilidad de acceder a algunas fuentes de importancia es factor que limita acceder a nuevas perspectivas. Entre las fuentes documentales, hasta el momento no ha sido posible acceder a los archivos de la AOA; respecto a las fuentes orales, dado el período analizado, pudimos localizar sólo una participante de las experiencias formativas de la década de 1950. Sin embargo, consideramos que es un valor de esta investigación haber logrado rescatar ese testimonio.

Por otra parte, en tanto estudio histórico, este no alcanza a describir el estado de relación actual entre la psicología y la odontología. Tampoco se caracteriza el lugar que ocupa el psicoanálisis hoy en la Argentina. Esto constituye una limitación, pero también señala vías de trabajo fructíferas para próximos desarrollos, entre cuyos propósitos podríamos incluir la descripción del estado actual de la relación interdisciplinaria psicología-odontología, así como la caracterización de los nuevos modelos de intervención. Sería sumamente valioso también 
aportar elementos que permitan valorar el papel actual de la psicología en la formación de odontólogos, y reconocer las razones del declive de la propuesta psicoanalítica tal como fue analizada en el presente estudio.

\section{Referencias}

Aberastury de Pichón-Riviere, A. (1951). Trastornos emocionales en el niño vinculados a la dentición. Revista Odontológica - Asociación Odontológica Argentina, 39(8), 357-359.

Aberastury, A. (1971). Aportaciones al psicoanálisis de niños. Buenos Aires: Paidós.

Aberastury, A. (1972). El psicoanálisis de niños y sus aplicaciones. Buenos Aires: Editorial Paidós.

Ardila, R. (1979). La psicología en Argentina: Pasado, presente, futuro. Revista Latinoamericana de Psicología, 11(1), 71-91.

Ardila, R. (2004). La psicología latinoamericana: El primer medio siglo. Interamerican Journal of Psychology, 38(2), 317-322.

Bloj, A. (2009). Arminda Aberastury: Pionera del psicoanálisis de niños en la Argentina. Norte de Salud Mental, 8(33), 13.

Bordoni, N. (2009). María Inés Egozcue y su visión anticipatoria: Centenario del nacimiento. Revista del Museo y Centro de Estudios Históricos, 41, 25.

Borinsky, M. (2006). Todo reside en saber qué es un niño: Aportes para una historia de la divulgación de las prácticas de crianza en la argentina. Anuario de investigaciones, 13, 117-126.

Buchbinder, P. (2005). Historia de las universidades argentinas. Buenos Aires: Sudamericana.

Carpintero, E., \& Vainer, A. (2004). Las huellas de la memoria: Psicoanálisis y salud mental en Argentina (Vol. 1). Buenos Aires: Ed. Topía.

Dagfal, A. (2009). Entre París y Buenos Aires: La invención del psicólogo (1942-1966). Buenos Aires: Paidós.

Danziger, K. (1979). Los orígenes sociales de la psicología moderna. En A. R. Buss (Ed.), Psychology in social context (H. A. Klappenbach, Trad., pp. 25-44). New York: Irvington Publishers.

Danziger, K. (1997). Naming the mind. London, UK: Sage Publications.

Delahanty, G. (2003). Vicisitudes de la polémica de Anna Freud y Melanie Klein. Revista Querencia, 6.

Easlick, K. A. (1935). The dentist's management of young children. International Journal of Orthodontia and Dentistry for Children, 21(1), 78-88.

Egozcue, M. I.; Manfredi, C.; \& Basso, M. L. (1988). Odontopediatría y psicología. Buenos Aires: Kargieman.

Falcone, R. (2010). Historia de la Psicología en la Argentina: Cruce de influencias europeas y carácter nacional. En Bicentenario: Ayer y hoy de la Psicologia Argentina (pp. 11-22). Buenos Aires: UCES.

Flick, U. (2004). Introducción a la investigación cualitativa. Madrid: Ediciones Morata.

Gacioppo, H. (1958). Programa de Odontopediatría. Facultad de Odontología. Córdoba: Universidad Nacional de Córdoba.

García, L., Macchioli, F., \& Talak, A. M. (2014). Psicología, niño y familia en la Argentina 19001970: Perspectivas históricas y cruces disciplinares. Buenos Aires: Biblos. Scherman 
Gesell, A., Ilg, F. L., Learned, J., \& Ames, L. B. (1943). Infant and child in the culture of today. Oxford, England: Harper.

Giordano, S. (2018). Programa de Psicología Evolutiva. Facultad de Odontología. Córdoba: Universidad Nacional de Córdoba.

Giordano, S., \& Alvárez, S. (2016). Historia de la Psicología en Córdoba: La relación Odontología y Psicología. Anuario Facultad de Psicología. Córdoba: Universidad Nacional de Córdoba.

Giordano, S., Scherman, P. \& Álvarez, P. S. (2017). Aportes de Arminda Aberastury a la práctica odontológica con niños. Repercusiones en Córdoba. Memorias IX Congreso Internacional de Investigación y Práctica Profesional en Psicología. Facultad de Psicología. Buenos Aires: Universidad de Buenos Aires. Recuperado de https://www.aacademica.org/000-067/163.pdf Gibbs, G. (2012). El análisis de datos en investigación cualitativa. Madrid: Ediciones Morata. Jacó-Vilela, A. M., Espírito-Santo, A. A., Degani-Carneiro, F., Oliveira, L., \& Vasconcellos, M. (2016). Investigando en historia de la psicología: Contribuciones metodológicas. Interacciones - Revista de Avances en Psicología, 2(2), 123-134.

Klappenbach, H. (2006). Periodización de la psicología en Argentina. Revista de Historia de la Psicología, 27(1), 109-164.

Klappenbach, H. (2014). Acerca de la Metodología de Investigación en la Historia de la Psicología. Psykhe, 23(1), 01-12.

Klein, M. (1944). La vida emocional y el desarrollo del yo del niño, con especial referencia a la posición depresiva. Traducido de The Freud-Klein Controversies.

Likierman, M. (1995). The debate between Anna Freud and Melanie Klein: An historical survey. Journal of Child Psychotherapy, 21(3), 313-325.

Moncunill de Chaer, I. (2012). Programa de Psicología Evolutiva. Facultad de Odontología. Córdoba: Universidad Nacional de Córdoba.

Neiburg, F. G., \& Plotkin, M. B. (2004). Intelectuales y expertos. Buenos Aires: Paidós.

Piñeda, M. A., \& Jacó-Vilela, A. M. (2014). Ciencia psicológica y profesionalización en Argentina y Brasil: 1930-1980. Universitas Psychologica, 13(5), 2015-2033.

Plotkin, M. B. (2003). Freud en las pampas: Orígenes y desarrollo de una cultura psicoanalítica en la Argentina (1910-1983). Buenos Aires: Sudamericana.

Rosa, A.; Huertas, J. A.; \& Blanco, F. (1996). Metodología para Historia de la Psicología. Madrid: Alianza.

Scherman, P. (2014). El ingreso del psicoanálisis en la argentina: Su influencia en la formación profesional del psicólogo. En H. Maldonado et al., Curso de Nivelación Manual 2015Psicología. 1a Ed. Córdoba: Universidad Nacional de Córdoba.

Universidad Nacional de Córdoba. (1956). Ordenanza de creación de la Facultad de Odontología. Córdoba: Universidad Nacional de Córdoba.

Universidad Nacional de Córdoba. (1985). Plan de Estudios de la Carrera de Odontología. Facultad de Odontología. Córdoba: Universidad Nacional de Córdoba.

Valles, M. S. (1999). Técnicas cualitativas de investigación social. Madrid: Síntesis Editorial. Vezzetti, H. (1996a). Los estudios históricos de la psicología en la Argentina. Cuadernos argentinos de historia de la psicología, 2(1-2), 79-93.

Vezzetti, H. (1996b). Aventuras de Freud en el país de los argentinos: De José Ingenieros a Enrique Pichon-Rivière. Buenos Aires: Paidós. 
Vezzetti, H. (2007). Historias de la psicología: Problemas, funciones y objetivos. Revista Historia de la Psicología, 28(1), 147-165.

Vilanova, A. (2003). Discusión por la psicología. Mar del Plata: UNMdP.

Watson, J. B. (1928). Psychological Care of Infant and Child.New York. US: W W Norton \& Co.

Watson, J. B. (1929). Behaviorist Psychology as Applied to the Child in the Dental Office. Oral Health.

Winkler, M. I., \& Wolff Reyes, X. (2005). El Buenos Aires kleiniano. Vida y obra de Arminda Aberastury (1910-1971). Acheronta, 22.

Recibido el: 28/07/2019

última revisión: 16/01/2020

Aceptación final: 11/02/2020

\section{Sobre las autoras:}

Patricia Scherman: Doctora en Psicología. Profesora titular de Sistemas de la Psicología Contemporánea. E-mail: patoscherman@gmail.com, Orcid: https://orcid.org/0000-0001-7340-1743

María Silvia Giordano: Licenciada en Psicología. Profesora adjunta a cargo de Psicología Evolutiva, Facultad de Odontología. E-mail: silvia_giordano@hotmail.com, Orcid: https://orcid.org/0000-0002-8085-8677 
